\title{
Tobacco-Derived Lipopolysaccharide, Not Microbial Translocation, as a Potential Contributor to the Pathogenesis of Rheumatoid Arthritis
}

\author{
Pieter W. A. Meyer $\mathbb{D}^{1,2}$ Mahmood M. T. M. Ally $\mathbb{D}^{1}{ }^{3}$ Mohammed Tikly $\mathbb{D}^{4},{ }^{4}$ \\ Gregory Tintinger, ${ }^{3}$ Lai Ling Winchow, ${ }^{4}$ Helen Steel $\left({ }_{0},{ }^{2}\right.$ and Ronald Anderson $\left(\mathbb{1}^{2}\right.$ \\ ${ }^{1}$ Department of Immunology, Tshwane Academic Division, National Health Laboratory Services, Pretoria 0001, South Africa \\ ${ }^{2}$ Department of Immunology, Faculty of Health Sciences, University of Pretoria, Pretoria 0001, South Africa \\ ${ }^{3}$ Department of Internal Medicine, Faculty of Health Sciences, University of Pretoria, Pretoria 0001, South Africa \\ ${ }^{4}$ Division of Rheumatology, Chris Hani Baragwaneth Academic Hospital, Faculty of Health Sciences, University of \\ the Witwatersand, Chris Hani Road, Johannesburg 2013, South Africa
}

Correspondence should be addressed to Pieter W. A. Meyer; pieter.meyer@up.ac.za

Received 19 August 2019; Accepted 4 October 2019; Published 3 November 2019

Academic Editor: Ronald Gladue

Copyright (c) 2019 Pieter W. A. Meyer et al. This is an open access article distributed under the Creative Commons Attribution License, which permits unrestricted use, distribution, and reproduction in any medium, provided the original work is properly cited.

\begin{abstract}
Microbial lipopolysaccharides (LPS) have been implicated in the pathogenesis of rheumatoid arthritis (RA), possibly driving a systemic inflammatory response that may trigger the development and/or exacerbation of the disease. To explore the existence of this mechanism in African RA patients, we have measured systemic levels of LPS and its surrogate, LPS-binding protein (LBP), as well as those of intestinal fatty acid-binding protein (I-FABP), pulmonary surfactant protein D (SP-D), and cotinine in serum to identify possible origins of LPS, as well as associations of these biomarkers with rheumatoid factor (RF) and anticitrullinated peptide (aCCP) autoantibodies and the DAS 28-3 clinical disease severity score. A cohort of 40 diseasemodifying antirheumatic drug-naïve, black South African RA patients rated by compound disease scores and 20 healthy subjects and 10 patients with chronic obstructive pulmonary disease (COPD) as controls were included in this study. Levels of the various biomarkers and autoantibodies were measured using a combination of ELISA and immunofluorimetric and immunoturbidometric procedures. LPS levels were lowest in the RA group compared to the healthy controls $(p=0.026)$ and COPD patients $(p=0.017)$, while LBP levels were also significantly lower in RA compared to the healthy individuals $(p=0.036)$. Levels of I-FABP and SP-D were comparable between all three groups. Categorisation of RA patients according to tobacco usage revealed the following significant positive correlations: LBP with C-reactive protein $(p=0.0137)$; a trend $(p=0.073)$ towards an association of LBP with the DAS 28-3 disease severity score; RF-IgG antibodies with both LPS and LBP $(p=0.033$ and $p=0.041$ , respectively); aCCP-IgG antibodies with LPS $(p=0.044)$; and aCCP-IgG with RF-IgM autoantibodies $(p=0.0016)$. The findings of this study, several of them novel, imply that tobacco products, as opposed to microbial translocation, represent a potential source of LPS in this study cohort of RA patients, again underscoring the risks posed by tobacco usage for the development and severity of RA.
\end{abstract}

\section{Introduction}

Evidence from earlier studies, both direct [1-5] and indirect [6-8], has implicated enteric bacteria and their proinflammatory products in the pathogenesis of rheumatoid arthritis (RA). However, it is only fairly recently that this concept has been revisited following the acquisition of insights into the role of the composition of the gut microbiome, as well as the structural integrity of the gut mucosa, in orchestrating immune responses [9-12]. This latter scenario involves leakage of bacterial products, especially lipopolysaccharides (LPS) and nucleic acids, from the gastrointestinal tract 
(GIT) and possibly from other anatomical sites, a process known as microbial translocation. These bacterial products, in turn, may trigger systemic, as well as localised, chronic inflammatory processes, especially in the lung and synovium, that may be linked to the development and perpetuation of RA [8]. In this context, it is noteworthy that bacterial LPS is known to initiate inflammatory mechanisms that cause protein citrullination [13], an event intimately linked to the immunopathogenesis of RA. In this context, autoantibodies to citrullinated proteins/peptides are not only diagnostic for RA but are also indicative of severe erosive disease [14-16]. Importantly, bacterial products are also present in cured tobacco [17-19], with smoking now well recognised as being a major risk factor for RA [20].

However, little is known about the possible involvement of microbial products in activating inflammatory mechanisms that may contribute to the pathogenesis of RA, particularly in the setting of the indigenous peoples of sub-Saharan Africa, who appear to experience a more severe form of disease [21-25]. To explore this issue in black South African RA patients, systemic concentrations of bacterial LPS and LPS-binding protein (LBP) were measured as indicators of endotoxaemia, while surfactant protein D (SP-D) and intestinal fatty acid-binding protein (I-FABP) were included as possible biomarkers of leakage from the lungs and GIT, respectively. LBP is a type 1 acute phase protein, similar to CRP and serum amyloid A (SAA), that recognises and binds the lipid A component of microbial LPS and is indicative of the host-defense response to proinflammatory endotoxins; SP-D is a collectin (a family of microbial pattern recognition receptors with opsonic properties) most commonly, but not exclusively, localised to the lungs, while I-FABP is expressed intracellularly in epithelial cells of the mucosa and is a systemic biomarker of epithelial leakage. Systemic concentrations of these various biomarkers of microbial translocation were then correlated with clinical and serological indices of disease activity.

\section{Materials and Methods}

The study cohort consisted of forty black South African RA patients recruited in 2013 from the rheumatology clinics of two tertiary academic hospitals in the Gauteng Province of South Africa. The patients complied with the 2010 ACR/EULAR RA criteria [26] and were disease-modifying antirheumatic drug- (DMARD-) naïve at baseline. Aliquots of serum prepared from coagulated blood were stored at $-80^{\circ} \mathrm{C}$ until measurement of the various test analytes described below. The control group consisted of 20 anonymous, matched, healthy blood donors (approval: 2017/18, South African National Blood Transfusion Service). To identify possible effects of chronic inflammatory lung disease on the test biomarkers, most importantly the utility of measurement of SP-D as a biomarker of pulmonary damage and leakage, an additional control group of 10 patients ( 5 female, 5 male) diagnosed with chronic obstructive pulmonary disease (COPD) was recruited from the Lung Unit, Department of Internal Medicine, Steve Biko Academic Hospital, Pretoria. They were all current or ex-tobacco users, and COPD was diagnosed by means of pulmonary function testing according to $\mathrm{FEV}_{1} / \mathrm{FVC}$ ratio of $<70 \%$. The mean postbronchodilator $\mathrm{FEV}_{1}$ was $69 \%$ of predicted, ranging from $36 \%$ of predicted (GOLD Stage III) to $102 \%$ of predicted (GOLD Stage I).

The study was approved by the Research Ethics Committee of the Faculty of Health Sciences of the University Pretoria, and informed, signed consent was obtained from all participants as required (approval number: 320/2016).

A chromogenic end-point limulus amoebocyte lysate method (LPS) (QCL-1000 ${ }^{\mathrm{TM}}$, Lonza Bioscience, Basel, Switzerland USA) was used to quantitate bacterial LPS with the results expressed as enzyme units $(\mathrm{EU}) / \mathrm{ml}$ serum, while LBP, SP-D, and I-FABP were assayed using ELISA procedures (all from Hycult Biotech, Netherlands) with the results expressed as nanograms (ng)/ml, micrograms $(\mu \mathrm{g}) / \mathrm{ml}$, and picograms $(\mathrm{pg}) / \mathrm{ml}$ serum, respectively. Serum cotinine levels were measured using an ELISA procedure (Calbiotech, Spring Valley, CA, USA) to verify the exposure to tobacco products in all groups, with results expressed as $\mathrm{ng} / \mathrm{ml}$ serum and a cut-off of $>5 \mathrm{ng} / \mathrm{ml}$ to identify those exposed to cigarettes, snuff, and other related tobacco products [27]. Anticitrullinated cyclic peptide (aCCP) IgG/IgA autoantibodies and rheumatoid factor (RF) $\operatorname{IgG} / \operatorname{IgA} / \operatorname{IgM}$ were determined using fluorimetric enzyme immunoassay procedures (EliA, Phadia AB, Oslo, Norway) with results expressed as autoantibody units (U)/ml serum, while high-sensitivity C-reactive protein (hs-CRP) was measured by laser nephelometry (Siemens AG, Munich, Germany) with results expressed as $\mu \mathrm{g} / \mathrm{ml}$ serum.

All RA patients were assessed clinically using several validated clinical disease scores (CDAI, SDAI, and DAS 28) [28, 29].

2.1. Statistical Analysis. Descriptive and inferential statistical procedures were used in the analysis of data. Tests for the association of contingency tables were performed using two-tailed chi ${ }^{2}$ tests. One-way ANOVA was performed using the Kruskal-Wallis test for nonparametric data for more than 2 groups or Dunn's test of multiple comparisons using rank sums. The Mann-Whitney test was applied when 2 groups were compared or the Wilcoxon test when the equality of matched pairs was determined. Correlation coefficients were derived from correlation matrices using the nonparametric Spearman's rank correlation test for multiple testing. The Bonferroni-Holm's correction was applied in the case of multiple correlations. Statistical significance was determined by a $p$ value $<0.05$ with statistical trending set at $p \leq 0.1$ to identify possible underlying significance. The analyses were done using Stata Statistical Software: Release 15 (StataCorp LLC, College Station, TX).

\section{Results and Discussion}

Demographic and duration of disease data, disease activity scores, and serum concentrations of hs-CRP, as well as those of anti-CCP and RF autoantibodies for the group of RA patients are shown in Table 1 . The median age and gender ratio $(\mathrm{F}: \mathrm{M})$ of the group of COPD control patients were 67 
TABLE 1: Demographics of RA patients.

\begin{tabular}{lccccc}
\hline & $N$ & Median & Iqr & Min & Max \\
\hline Age (Yr) & 40 & 54.5 & 19 & 19 & 79 \\
CDAI & 40 & 23.8 & 19.8 & 6 & 55 \\
DAS 28-3 & 40 & 4.8 & 1.4 & 2.9 & 5 \\
SDAI & 40 & 26.4 & 18.8 & 7.7 & 60 \\
hs-CRP & 40 & 12 & 26.5 & 1 & 116 \\
Dur (m) & 40 & 7 & 5 & 0 & 14 \\
RF-IgM & 40 & 46 & 92,5 & 1.8 & 2857 \\
RF-IgA & 40 & 20.0 & 41.5 & 4.1 & 395 \\
aCCP-IgG & 40 & 166 & 441.5 & 0.6 & 1652 \\
aCCP-IgA & 40 & 9.8 & 33.8 & 0.9 & 108 \\
\hline
\end{tabular}

Yr-years; CDAI-Clinical Disease Activity Index; DAS-disease activity score; SDAI-Simplified Disease Activity Index; hs-CRP—high-sensitivity $\mathrm{C}$ reactive protein; Dur $(\mathrm{m})$-disease duration in months; $\mathrm{RF}$-rheumatoid factor; aCCP—anticyclic citrullinated peptides; Ig-immunoglobulin.

TABLE 2: Cotinine levels ( $\mathrm{ng} / \mathrm{ml}$ ) of the RA and control groups.

\begin{tabular}{lcccccc}
\hline & \multicolumn{2}{c}{ RA } & \multicolumn{2}{c}{ COPD } & \multicolumn{2}{c}{ Healthy } \\
& NTU & TU & NTU & TU & NTU & TU \\
\hline$N$ & 24 & 16 & 6 & 4 & 8 & 12 \\
Median & 0 & 77 & 0 & 76 & 0 & 95 \\
Iqr & 0 & 94 & 0 & 75 & 0 & 6 \\
Min & 0 & 5 & 0 & 17 & 0 & 93 \\
Max & 0 & 133 & 0 & 133 & 0 & 103 \\
\hline
\end{tabular}

COPD—chronic obstructive pulmonary disease; RA-rheumatoid arthritis; NTU—nontobacco usage; TU—-tobacco usage; Iqr-interquartile range.

years and $1: 1$, respectively. Serum cotinine levels of the different groups are shown in Table 2.

The concentrations of the four test biomarkers of microbial translocation, LPS, SP-D, I-FABP, and LBP for the group of RA patients and both control groups are shown in Figures $1(\mathrm{a})-1(\mathrm{~d})$. Serum concentrations of LPS were significantly lower in the RA group relative to both the healthy $(p=0.026)$ and the COPD $(p=0.017)$ control groups following application of Dunn's post hoc test. Median levels of LBP were significantly different in all groups $(p=0.002)$, as well as between RA and healthy $(p=0.036)$ and RA and COPD patients $(p=0.001)$. With respect to SP-D and I-FABP, serum levels of both of these biomarkers failed to attain statistical significance.

3.1. Multiple Pairwise Correlations between the Test Biomarkers, as well as Correlations of These with Indicators of Disease Activity in RA Patients. A weak positive correlation was noted between hs-CRP and LBP $(r=0.3812 ; p=0.0152)$ in the entire cohort of RA patients, which was strongest in the subgroup of users of tobacco products $(r=0.6018$; $p=0.0137)$. LBP also showed a trend towards correlating with DAS 28-3 in the subgroup of RA patients that used tobacco products $(r=0.4599 ; p=0.0731)$, while a moderate correlation between serum SP-D and disease duration $(r=0.4181 ; p=0.0073)$, as well as weak negative correlations of this biomarker with I-FABP $(r=-0.2869$; $p=0.0123)$ and LPS $(r=-0.3287 ; p=0.0411)$ were also noted.

3.2. Multiple Pairwise Correlations of Autoantibodies with Endotoxin, $L B P, S P-D$, and I-FABP. These results are shown in Table 3. The most notable positive correlations were observed in the subgroup of tobacco users between (i) LPS and RF IgG autoantibodies $(r=0.53 ; p=0.033)$, (ii) LBP and aCCP IgG autoantibodies $(r=0.53 ; p=0.044)$, and (iii) LBP and RF IgG antibodies $(r=0.51 ; p=0.04)$. No statistically significant associations of hs-CRP with either aCCP or $\mathrm{RF}$ autoantibodies were detected. Interestingly, a statistically significant negative correlation was detected between SP-D and CCP IgG antibodies in the subgroup of tobacco users $(r=-0.53 ; p=0.035)$. These observations clearly underscore the positive relationships between LPS and elevations in systemic biomarkers of RA.

Wilcoxon's matched-pairs signed-ranks testing of autoantibody isotypes in RA, these being aCCP-IgG and RF-IgM, as included in the ACR/EULAR 2010 scoring criteria for RA diagnosis, revealed that aCCP-IgG and RF-IgM were statistically significantly different ( $Z$ score $=2.379$, $p=0.0174$ ), meaning that a highly elevated aCCP-IgG titre in a patient did not necessarily correlate with a highly elevated RF-IgM titre. This finding was consistent across the other isotypes tested ( $Z$ score range $=-3.76-4.28, p$ value range $=0.001-0.042)$. However, when categorised according to tobacco use, a strong, statistically significant correlation between concentrations of aCCP and RF autoantibodies of the IgG isotype was detected $(r=0.72 ; p=0.0016)$ in the subgroup of RA tobacco users.

\section{Discussion}

A number of publications have alluded to the possible involvement of the intestinal and pulmonary microbiota, as well as environmental, lifestyle, and occupational factors, in triggering or exacerbating the development of RA in both humans and in murine models of experimental disease $[11,12,20,30-36]$. However, the potential role of proinflammatory bacterial products, especially endotoxins, reactive with pathogen recognition receptors on cells of the innate immune system, in the immunopathogenesis of RA, remains largely uncertain. This is particularly the case in sub-Saharan Africa, a geographic region where poverty and ongoing urbanisation have been accompanied by an increased prevalence of RA [37], often presenting as severe disease $[21-25,38]$.

However, in the current study, levels of circulating LPS were found to be significantly lower in the group of RA patients when compared with those of both the healthy and COPD control groups, while levels of I-FABP were comparable between the groups, seemingly excluding leakage of LPS from the GIT. Serum concentrations of the other two biomarkers, SP-D and LBP, were also comparable in all three groups. With respect to associations between isotypes of RF and aCCP autoantibodies in the entire group of RA patients, 


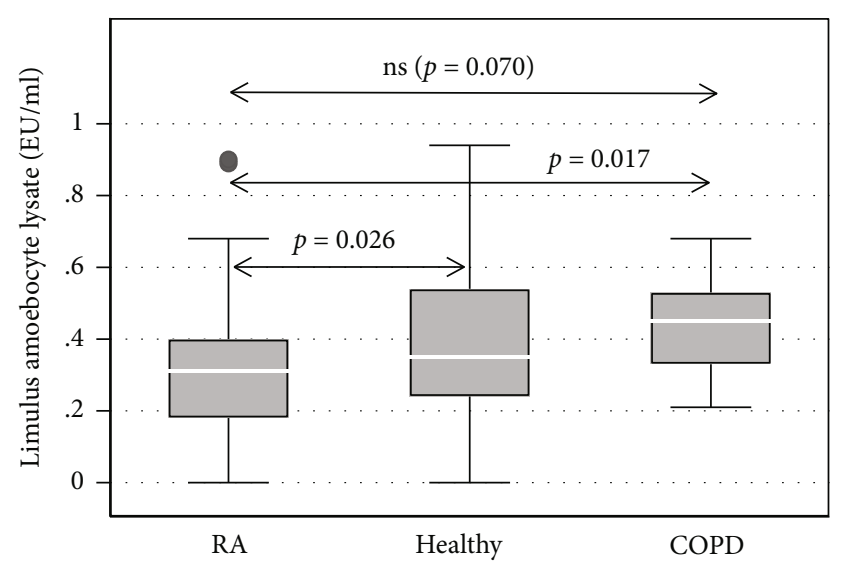

(a)

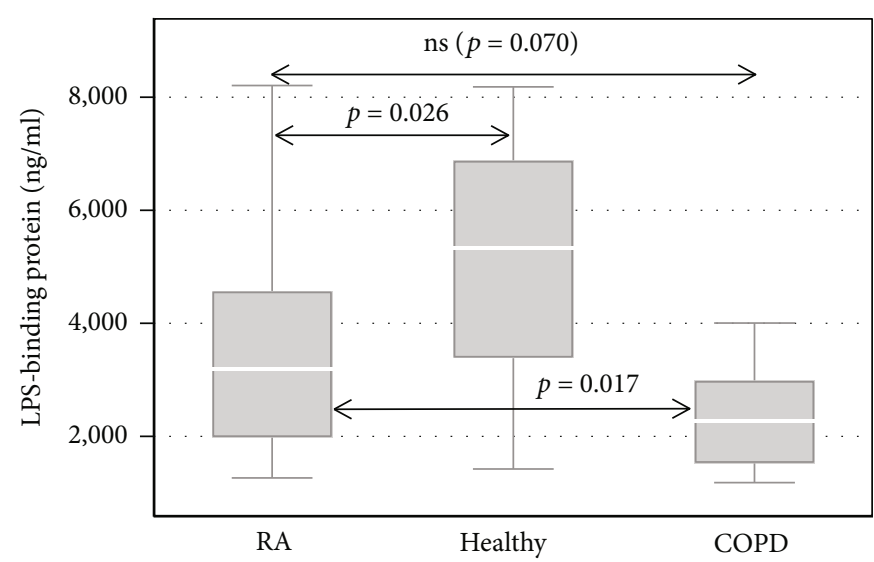

(b)

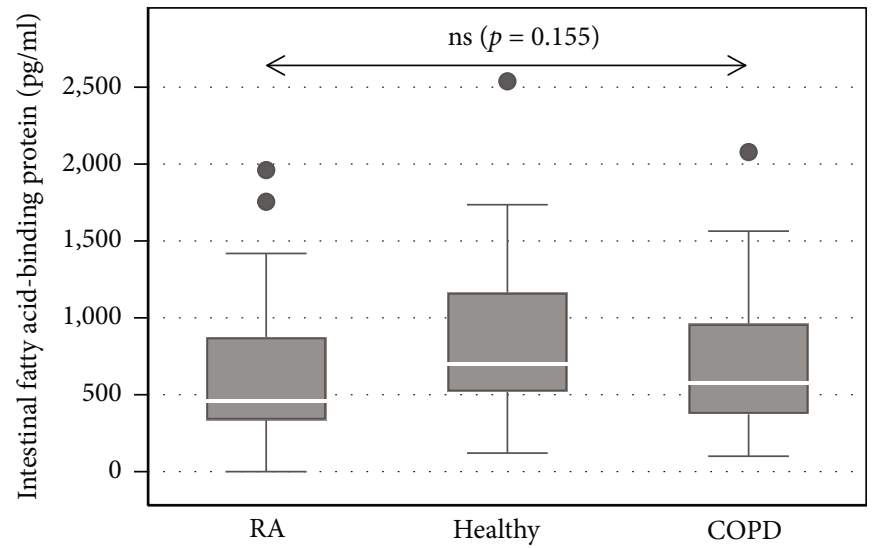

(c)

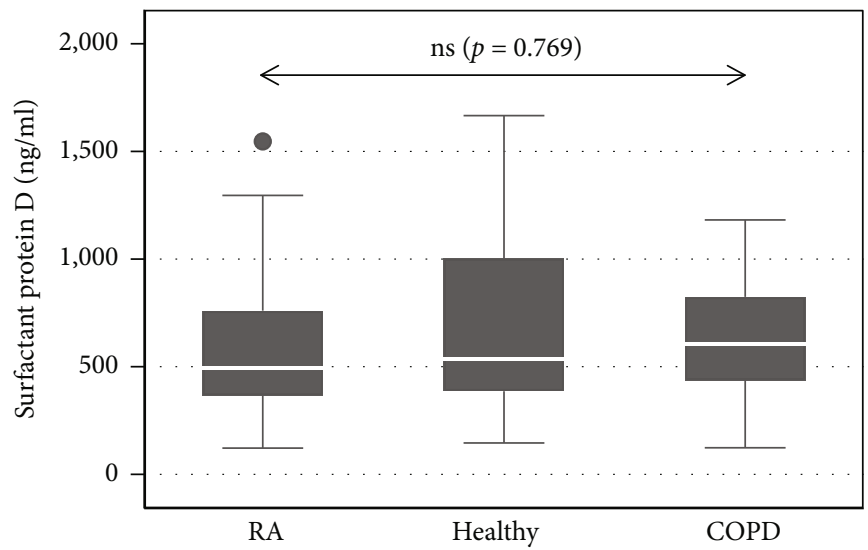

(d)

FIGURE 1: Box and whisker graphs showing median concentrations of the four biomarkers and statistical differences between the groups as per Dunn's post hoc test.

weak, albeit statistically significant, positive correlations were detected between LPS and aCCP-IgG, as well as between LBP and RF-IgG. LBP also showed a weak, positive, significant correlation with hs-CRP, which is not surprising since both biomarkers function as positive acute phase reactants. A trend towards a positive association between LBP and DAS 28-3 scores was also noted in RA patients exposed to tobacco products, as previously reported by Wen et al. [10]. Although interesting, the slightly lower, albeit statisti- cally significant, levels of circulating LPS observed in the overall group of RA patients relative to the control groups are somewhat surprising and difficult to explain. One possibility, albeit speculative, is that the RA-associated chronic inflammatory response results in accelerated hepatic and/or antibody-mediated clearance of LPS. Alternatively, this discrepancy may result from the lower prevalence of tobacco usage in the RA group relative to the control group in particular. 
TABLE 3: Correlations between disease indicators and the various test biomarkers.

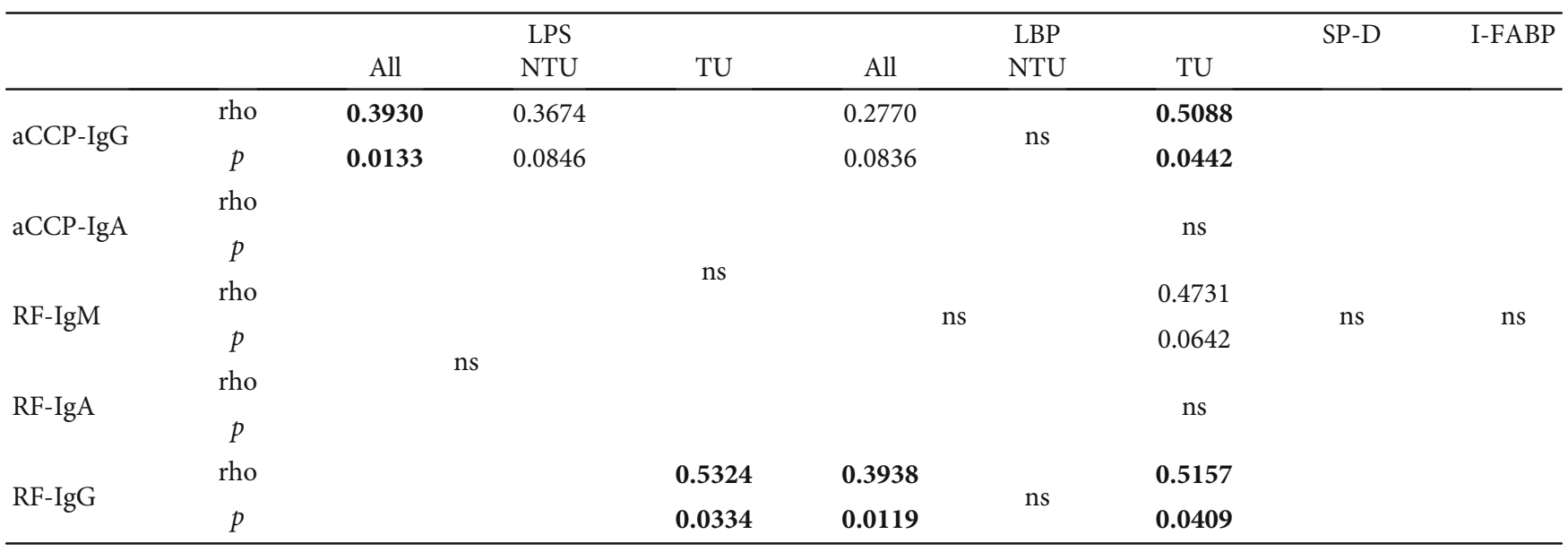

Key: aCCP-anticyclic citrullinated peptide; RF-rheumatoid factor; Ig-immunoglobulin; LPS-lipopolysaccharide; LBP_LPS-binding protein; SP-D—surfactant protein D; I-FABP—intestinal fatty acid-binding protein; NTU—nontobacco usage; TU—-tobacco usage; ns—not significant.

Despite the apparent lack of associations between circulating LPS and indices of disease activity in the entire group of patients with RA noted in the current study, categorisation of the patients according to tobacco usage proved to be revealing. Statistically significant associations, two novel and one previously described, of smoking with inflammatory biomarkers were identified, further underscoring the wellrecognised risks posed by tobacco usage to those with both developing and existing disease. The novel positive associations were those between (i) LPS, as well as its surrogate, LBP, with RF IgG autoantibodies and (ii) LBP with aCCP IgG autoantibodies. The strongly significant positive association between smoking and elevated serum levels of both aCCP and RF autoantibodies detected in the current study has been described previously [39]. A fourth association, a statistically significant negative relationship between serum SP-D and aCCP may seem surprising. However, this may be explained by a finding that smoking, which triggers protein citrullination in the lungs [40,41], is associated with significantly decreased pulmonary concentrations of SP-D [42].

In the context of the aforementioned findings, it is noteworthy that usage of nasally inhaled snuff, as opposed to cigarette smoking, is common practice among older African females [27], with both types of tobacco usage resulting in similar levels of serum cotinine [43]. Given that tobacco contains high concentrations of LPS [17-19], it is therefore possible that exposure to tobacco-derived bacterial products, as opposed to microbial translocation from the GIT and/or lungs, may account for the associations of LPS/LBP with both types of autoantibodies, as well as for the strong positive interrelationship between aCCP and RF autoantibodies. Moreover, LBP may represent a more accurate indicator of LPS-induced inflammatory stress, possibly due to the instability of, and or complex-forming by, LPS.

\section{Conclusions}

The findings of this study imply that exposure to tobacco products, as opposed to microbial translocation, represents the probable source of LPS in our cohort of RA patients, a contention that is based on the detection of associations, several of which are novel, between cotinine levels, LPS, LBP, and RA-associated autoantibodies.

\section{Data Availability}

The numeric data used to support the findings of this study are available from the corresponding author upon request.

\section{Conflicts of Interest}

The authors declare that there is no conflict of interest regarding the publication of this paper.

\section{Acknowledgments}

The research and publication of this article is funded by departmental research funds.

\section{References}

[1] P. Wagener, J. Busch, M. Hammer, and R. Brunkhorst, "Endotoxin in the plasma of patients with inflammatory rheumatic diseases," Scandinavian Journal of Rheumatology, vol. 17, no. 4, pp. 301-303, 1988.

[2] O. Maki-Ikola and K. Granfors, "Salmonella-triggered reactive arthritis,” The Lancet, vol. 339, no. 8801, pp. 1096-1098, 1992.

[3] D. Heumann, S. Bas, P. Gallay et al., "Lipopolysaccharide binding protein as a marker of inflammation in synovial fluid of patients with arthritis: correlation with interleukin 6 and C-reactive protein," The Journal of Rheumatology, vol. 22, no. 7, pp. 1224-1229, 1995.

[4] J. H. Lange, "Endotoxin as a factor for joint pain and rheumatoid arthritis," Clinical Rheumatology, vol. 23, no. 6, p. 566, 2004.

[5] Z. Y. Huang, T. Stabler, F. X. Pei, and V. B. Kraus, "Both systemic and local lipopolysaccharide (LPS) burden are associated with knee OA severity and inflammation," Osteoarthritis and Cartilage, vol. 24, no. 10, pp. 1769-1775, 2016. 
[6] S. Aoki, K. Yoshikawa, T. Yokoyama et al., "Role of enteric bacteria in the pathogenesis of rheumatoid arthritis: evidence for antibodies to enterobacterial common antigens in rheumatoid sera and synovial fluids," Annals of the Rheumatic Diseases, vol. 55, no. 6, pp. 363-369, 1996.

[7] M. S. Di Genaro, E. Muñoz, C. Aguilera, and A. M. S. de Guzmán, "Yersinia enterocolitica O:8 and O:5 lipopolysaccharide arthritogenicity in hamsters," Rheumatology, vol. 39, no. 1, pp. 73-78, 2000.

[8] W. Lorenz, C. Buhrmann, A. Mobasheri, C. Lueders, and M. Shakibaei, "Bacterial lipopolysaccharides form procollagen-endotoxin complexes that trigger cartilage inflammation and degeneration: implications for the development of rheumatoid arthritis," Arthritis Research \& Therapy, vol. 15, no. 5, p. R111, 2013.

[9] L. J. Kasselman, N. A. Vernice, J. Deleon, and A. B. Reiss, “The gut microbiome and elevated cardiovascular risk in obesity and autoimmunity," Atherosclerosis, vol. 271, pp. 203-213, 2018.

[10] W. Wen, Y. Li, Y. Cheng et al., "Lipopolysaccharide-binding protein is a sensitive disease activity biomarker for rheumatoid arthritis," Clinical and Experimental Rheumatology, vol. 36, no. 2, pp. 233-240, 2018.

[11] A. I. Catrina, K. D. Deane, and J. U. Scher, "Gene, environment, microbiome and mucosal immune tolerance in rheumatoid arthritis," Rheumatology, vol. 55, no. 3, pp. 391-402, 2014.

[12] Y. Jeong, J.-W. Kim, H. J. You et al., "Gut microbial composition and function are altered in patients with early rheumatoid arthritis," Journal of Clinical Medicine, vol. 8, no. 5, p. 693, 2019.

[13] E. Darrah and F. Andrade, "Rheumatoid arthritis and citrullination," Current Opinion in Rheumatology, vol. 30, no. 1, pp. 72-78, 2018.

[14] A. A. Jilani and C. G. Mackworth-Young, "The role of citrullinated protein antibodies in predicting erosive disease in rheumatoid arthritis: a systematic literature review and meta-analysis," International Journal of Rheumatology, vol. 2015, Article ID 728610, 8 pages, 2015.

[15] W. Li, E. H. Sasso, A. H. M. van der Helm-van Mil, and T. W. J. Huizinga, "Relationship of multi-biomarker disease activity score and other risk factors with radiographic progression in an observational study of patients with rheumatoid arthritis," Rheumatology, vol. 55, no. 2, pp. 357-366, 2016.

[16] P. W. A. Meyer, M. T. M. Ally, B. Hodkinson, R. Anderson, and M. Tikly, "Comparison of the diagnostic potential of three anti-citrullinated protein antibodies as adjuncts to rheumatoid factor and CCP in a cohort of South African rheumatoid arthritis patients," Rheumatology International, vol. 38, no. 6 , pp. 993-1001, 2018.

[17] J. D. Hasday, R. Bascom, J. J. Costa, T. Fitzgerald, and W. Dubin, "Bacterial endotoxin is an active component of cigarette smoke," Chest, vol. 115, no. 3, pp. 829-835, 1999.

[18] L. Larsson, B. Szponar, B. Ridha et al., "Identification of bacterial and fungal components in tobacco and tobacco smoke," Tobacco Induced Diseases, vol. 4, no. 1, p. 4, 2008.

[19] A. R. Sapkota, S. Berger, and T. M. Vogel, "Human pathogens abundant in the bacterial metagenome of cigarettes," Environmental Health Perspectives, vol. 118, no. 3, pp. 351-356, 2010.

[20] R. Anderson, P. W. Meyer, M. M. Ally, and M. Tikly, "Smoking and air pollution as pro-inflammatory triggers for the development of rheumatoid arthritis," Nicotine \& Tobacco Research, vol. 18, no. 7, pp. 1556-1565, 2016.

[21] P. W. A. Meyer, S. W. Brighton, and R. Anderson, “Association of the HLA-DRB $1 * 04$ allele and its subtypes with rheumatoid arthritis in South Africa," South African Journal of Science, vol. 100, no. 5, pp. 305-306, 2004.

[22] B. Hodkinson, E. Musenge, M. Ally, P. W. A. Meyer, R. Anderson, and M. Tikly, "Response to traditional diseasemodifying anti-rheumatic drugs in indigent South Africans with early rheumatoid arthritis," Clinical Rheumatology, vol. 31, no. 4, pp. 613-619, 2012.

[23] P. W. A. Meyer, B. Hodkinson, M. Ally et al., "HLA-DRB1 shared epitope genotyping using the revised classification and its association with circulating autoantibodies, acute phase reactants, cytokines and clinical indices of disease activity in a cohort of South African rheumatoid arthritis patients," Arthritis Research \& Therapy, vol. 13, no. 5, article ar3479, p. R160, 2011.

[24] B. Hodkinson, E. Musenge, M. Ally, P. Meyer, R. Anderson, and M. Tikly, "Functional disability and health-related quality of life in South Africans with early rheumatoid arthritis," Scandinavian Journal of Rheumatology, vol. 41, no. 5, pp. 366-374, 2012.

[25] M. M. T. M. Ally, P. W. A. Meyer, and R. Anderson, "Early rheumatoid arthritis: focus on RA in the developing world," South African Family Practice, vol. 58, no. 4, pp. 164-166, 2016.

[26] J. Kay and K. S. Upchurch, "ACR/EULAR 2010 rheumatoid arthritis classification criteria," Rheumatology, vol. 51, Supplement 6, pp. vi5-vi9, 2012.

[27] N. L. Benowitz, J. T. Bernert, R. S. Caraballo, D. B. Holiday, and J. Wang, "Optimal serum cotinine levels for distinguishing cigarette smokers and nonsmokers within different racial/ethnic groups in the United States between 1999 and 2004," American Journal of Epidemiology, vol. 169, no. 2, pp. 236-248, 2009.

[28] M. L. L. Prevoo, M. A. Van'T Hof, H. H. Kuper, M. A. Van Leeuwen, L. B. A. Van De Putte, and P. L. C. M. Van Riel, "Modified disease activity scores that include twenty-eightjoint counts development and validation in a prospective longitudinal study of patients with rheumatoid arthritis," Arthritis and Rheumatism, vol. 38, no. 1, pp. 44-48, 1995.

[29] D. Aletaha and J. Smolen, "The Simplified Disease Activity Index (SDAI) and the Clinical Disease Activity Index (CDAI): a review of their usefulness and validity in rheumatoid arthritis," Clinical and Experimental Rheumatology, vol. 23, no. 5, pp. S100-S108, 2005.

[30] H. T. Hogenová, R. Štěpánková, T. Hudcovic et al., "Commensal bacteria (normal microflora), mucosal immunity and chronic inflammatory and autoimmune diseases," Immunology Letters, vol. 93, no. 2-3, article S0165247804000379, pp. 97-108, 2004.

[31] J. U. Scher and S. B. Abramson, "The microbiome and rheumatoid arthritis," Nature Reviews Rheumatology, vol. 7, no. 10, pp. 569-578, 2011.

[32] A. D. Proal, P. J. Albert, and T. G. Marshall, "The human microbiome and autoimmunity," Current Opinion in Rheumatology, vol. 25, no. 2, pp. 234-240, 2013.

[33] V. Taneja, "Arthritis susceptibility and the gut microbiome," FEBS Letters, vol. 588, no. 22, pp. 4244-4249, 2014.

[34] L. Allais, S. Kumar, K. Debusschere et al., "The effect of cigarette smoke exposure on the development of inflammation in 
lungs, gut and joints of TNF $\triangle \mathrm{ARE}$ mice," PLoS One, vol. 10, no. 11, article e0141570, 2015.

[35] J. U. Scher, V. Joshua, A. Artacho et al., “The lung microbiota in early rheumatoid arthritis and autoimmunity," Microbiome, vol. 4, no. 1, article 206, p. 60, 2016.

[36] P. M. Wells, F. M. K. Williams, M. L. Matey-Hernandez, C. Menni, and C. J. Steves, "RA and the microbiome: do host genetic factors provide the link?," Journal of Autoimmunity, vol. 99, pp. 104-115, 2019.

[37] G. M. Mody, "Rheumatology in Africa-challenges and opportunities," Arthritis Research \& Therapy, vol. 19, no. 1, p. $49,2017$.

[38] M. Niasse, B. S. Kane, A. A. Ndiaye et al., "Severity of the rheumatoid arthritis in sub-Saharan Africa: study of 403 Senegalese observations," Open Journal of Internal Medicine, vol. 7, no. 4, pp. 151-159, 2017.

[39] A. Krol, P. Garred, N. H. Heegaard et al., "Interactions between smoking, increased serum levels of anti-CCP antibodies, rheumatoid factors, and erosive joint disease in patients with early, untreated rheumatoid arthritis," Scandinavian Journal of Rheumatology, vol. 44, no. 1, pp. 8-12, 2015.

[40] L. Klareskog, P. Stolt, K. Lundberg et al., "A new model for an etiology of rheumatoid arthritis: Smoking may trigger HLA-DR (shared epitope)-restricted immune reactions to autoantigens modified by citrullination," Arthritis and Rheumatism, vol. 54, no. 1, pp. 38-46, 2006.

[41] D. Makrygiannakis, M. Hermansson, A. K. Ulfgren et al., "Smoking increases peptidylarginine deiminase 2 enzyme expression in human lungs and increases citrullination in BAL cells," Annals of the Rheumatic Diseases, vol. 67, no. 10, pp. 1488-1492, 2008.

[42] J. M. More, D. R. Voelker, L. J. Silveira, M. G. Edwards, E. D. Chan, and R. P. Bowler, "Smoking reduces surfactant protein $\mathrm{D}$ and phospholipids in patients with and without chronic obstructive pulmonary disease," BMC Pulmonary Medicine, vol. 10, no. 1, article 223, p. 53, 2010.

[43] E. R. Gritz, V. Baer-Weiss, N. L. Benowitz, H. Van Vunakis, and M. E. Jarvik, "Plasma nicotine and cotinine concentrations in habitual smokeless tobacco users," Clinical Pharmacology and Therapeutics, vol. 30, no. 2, pp. 201-209, 1981. 


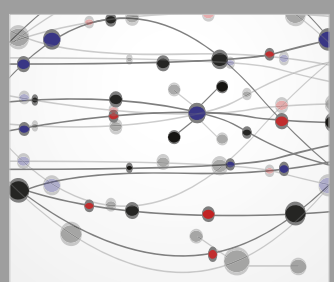

The Scientific World Journal
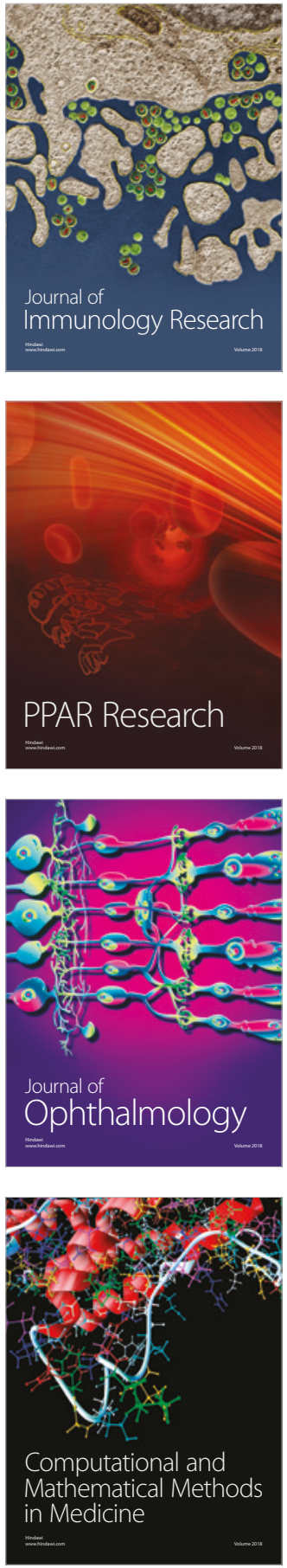

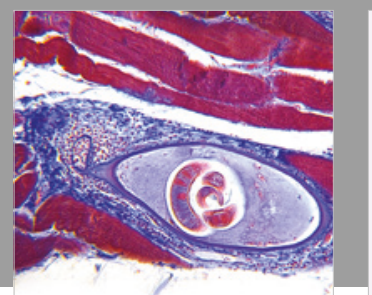

Gastroenterology Research and Practice

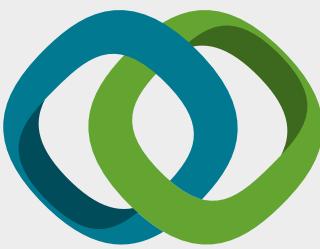

\section{Hindawi}

Submit your manuscripts at

www.hindawi.com
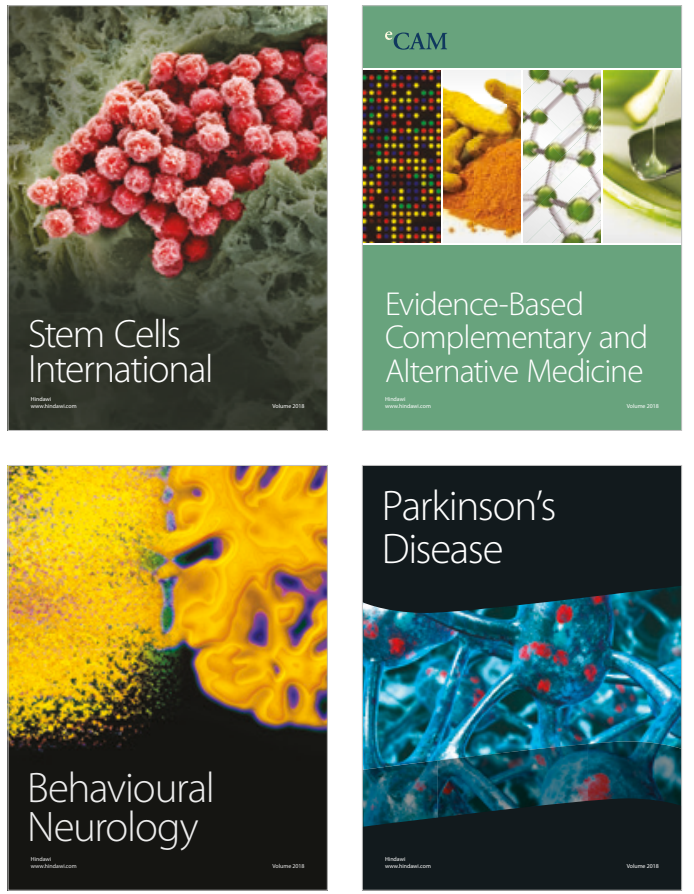

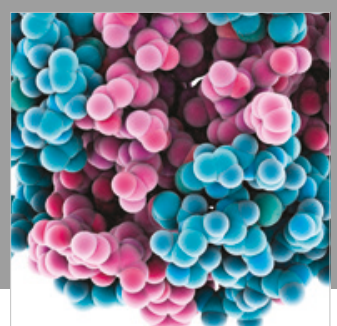

ournal of

Diabetes Research

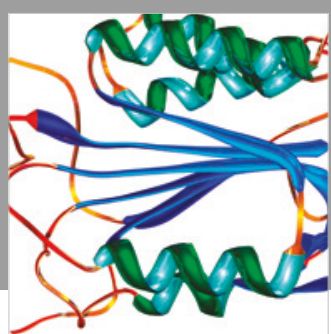

Disease Markers
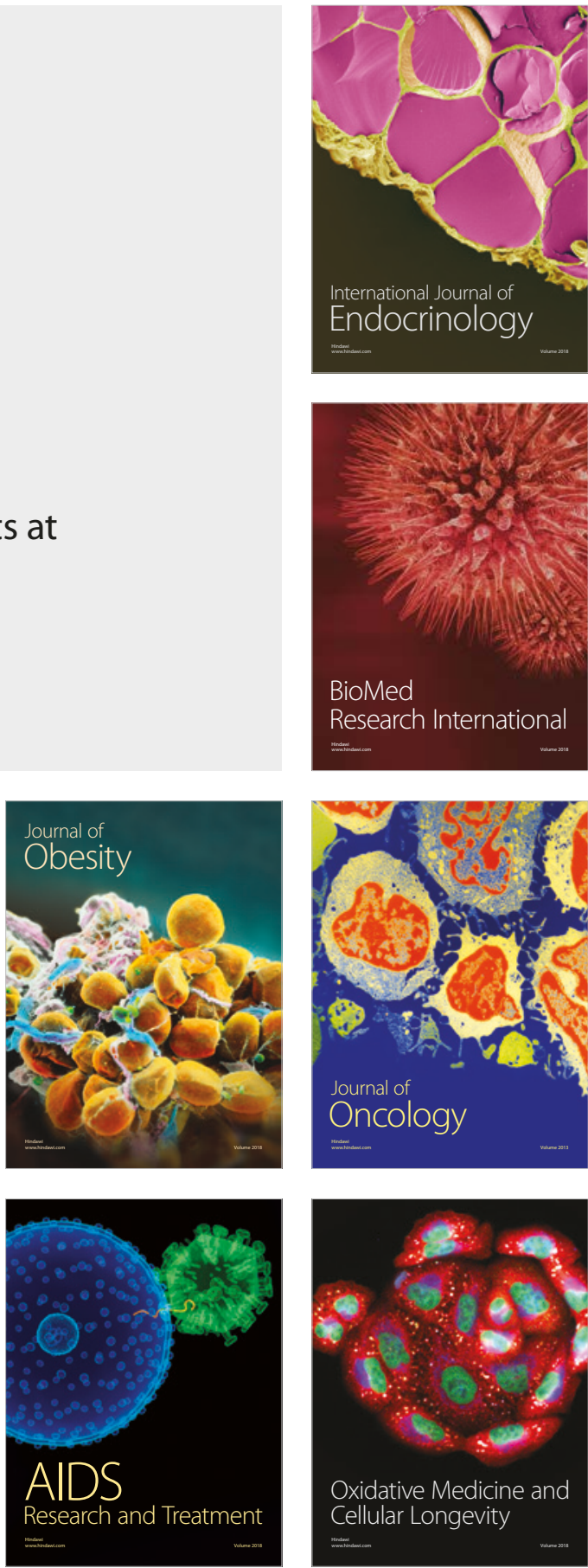